\title{
Seven Components of Judgmental Forecasting Skill: Implications for Research and the Improvement of Forecasts
}

\author{
THOMAS R. STEWART \\ Center for Policy Research, University at Albany, State University \\ of New York, U.S.A. \\ and
}

CYNTHIA M. LUSK

Center for Research on Judgment and Policy, University of Colorado, U.S.A.

\begin{abstract}
A decomposition of the Brier skill score shows that the performance of judgmental forecasts depends on seven components: environmental predictability, fidelity of the information system, match between environment and forecaster, reliability of information acquisition, reliability of information processing, conditional bias, and unconditional bias. These components provide a framework for research on the forecasting process. Selected literature addressing each component is reviewed, and implications for improving judgmental forecasting are discussed.
\end{abstract}

KEY WORDS Judgmental forecasting Brier skill score Lens model equation Bias Reliability

In any field requiring judgmental forecasts, the performance of professional forecasters depends jointly on (1) the environment about which forecasts are made, (2) the information system that brings data about the environment to the forecaster, and (3) the cognitive system of the forecaster. For example, in weather forecasting the environment includes the atmosphere and the land, ocean and solar features that affect weather. The information system includes the instruments, observations, and algorithms that produce information about past and current weather and the communication and display systems that bring that information to the forecaster. The cognitive system consists of the perceptual and judgmental processes that the forecaster uses to acquire information, aggregate it, and produce the forecast.

This paper describes how certain properties of these three systems combine to determine forecasting performance. A commonly used measure of skill (the 'skill score' based on the mean-square-error) is analyzed into seven components. Since each component describes a different aspect of forecast performance, the decomposition suggests a framework for research on judgmental forecasting. 
The next section describes the basic measure of forecasting skill adopted in this paper. Then a decomposition of that measure recently developed by Allan Murphy is described. In the following sections, a further decomposition into seven components based on the "lens model equation' is developed. Finally, selected research on each of the seven components and implications for improving forecasting skill is reviewed.

Although this paper focuses on judgmental forecasting, much of the material discussed is equally applicable to other types of professional judgment (e.g. diagnostic judgments made by physicians). Furthermore, the decomposition we propose can be applied to either probabilistic or deterministic judgments, and we have relied on the literature describing both types of judgment where appropriate.

\section{MEASUREMENT OF FORECASTING PERFORMANCE}

The most commonly used measures of forecasting performance can be roughly classified into three main groups: (1) those based on mean-square-error, (2) those based on correlation, and (3) those based on signal detection theory (SDT). The decomposition used in this paper combines measures from the first two groups. Although SDT is a powerful and elegant technique (Harvey et al., 1992), we did not find it suitable for our analysis because no decomposition comparable in analytic power to the lens model equation (described below) is available in the SDT framework. Furthermore, the measures provided by SDT are independent of biases in the means or standard deviations of forecasts. Since those biases have been the focus of a substantial body of research and they can be important to the users of forecasts, it is important to include them in any formulation that purports to describe forecasting performance. It should also be noted that there is a strong conceptual similarity between the SDT model and the other two types of measures (Yates and Curley, 1985, p. 69).

The mean-square-error (MSE) is defined as:

$$
\operatorname{MSE}_{Y}=\left(\frac{1}{n}\right) \Sigma\left(Y_{i}-O_{i}\right)^{2}
$$

where $n$ is the number of forecasts, $Y_{i}$ is the $i$ th forecast, and $O_{i}$ is the observed value corresponding to that forecast.

The skill of a forecast is measured by comparing its MSE with that of a reference forecast. One possible reference is a constant forecast of the mean of the variable being forecast. The MSE for such a reference forecast would be:

$$
\operatorname{MSE}_{B}=\left(\frac{1}{n}\right) \Sigma\left(\bar{O}-O_{i}\right)^{2}
$$

where $\bar{O}$ is the mean of the observed events in the sample. A skill score is then defined by:

$$
\mathrm{SS}=1-\left(\frac{\mathrm{MSE}_{Y}}{\mathrm{MSE}_{B}}\right)
$$

For both probabilistic and deterministic forecasts, this skill score is 1.0 for perfect forecasts, 0.0 if the forecast is only as accurate as the reference forecast, and negative if the forecast is less accurate than the reference forecast. Although not the only possible choice of a reference forecast (Murphy, 1988), the sample mean permits a decomposition of skill that is simple, elegant, and useful for understanding the components of skill. 


\section{DECOMPOSITION OF SKILL}

This section describes in three steps the logic for decomposing the skill score into seven components.

\section{Step 1: Murphy's decomposition}

A variety of decompositions of MSE and skill scores have been developed (Cronbach, 1955; Sanders, 1963; Murphy, 1972a,b, 1973, 1988; Theil, 1966; Yates, 1982; Lee and Yates, 1992). We have used a decomposition developed by Murphy (1988) because it most clearly shows the relation between the correlation coefficient and the skill score. Murphy showed that the skill score can be decomposed as follows:

$$
\mathrm{SS}=\left(r_{Y O}\right)^{2}-\left[r_{Y O}-\left(\frac{s_{Y}}{s_{O}}\right)\right]^{2}-\left[\frac{(\bar{Y}-\bar{O})}{s_{O}}\right]^{2}
$$

where $r_{Y O}$ is the correlation between the forecast and the observed event; $s_{Y}$ and $s_{O}$ are the standard deviations of the forecast and the observed event, respectively; and $\bar{Y}$ and $\bar{O}$ are the means of the forecast and the observed event.

The first term in Murphy's decomposition is the squared correlation coefficient. For probability forecasts, the correlation is closely related to the 'resolution' of forecasts (Sanders, 1963; Murphy, 1973). Resolution reflects 'the ability to discriminate occasions when event A will and will not take place' (Yates, 1982, p. 136). The correlation is equal to the skill score only when the second and third terms, which measure forecast bias, are zero. Murphy (1988) stated that the correlation can be considered a measure of 'potential' skill in the forecast; that is, the skill that the forecaster could obtain by eliminating bias from the forecasts.

Murphy called the second term 'conditional bias'. It is zero when the slope of the regression line for predicting observed events from forecasts is 1.0. This term could also be called 'regression bias' because it indicates whether the standard deviations of the forecasts are appropriately reduced to account for a less than perfect correlation. When the correlation is low, the variability of the forecasts, as measured by their standard deviation, must be reduced in order to maintain a slope of 1.0. If regression analysis were used to make forecasts, this reduction would occur automatically (Stewart and Reagan-Cirincione, 1991) and conditional bias would be zero.

Murphy called the third term 'unconditional bias'. It reflects the match between the mean of the forecasts and the mean of the observations. As the difference between these means increases, the intercept of the regression line departs from zero. For probability forecasts, this term of Murphy's decomposition could be called 'base rate bias' because it measures how well the mean forecast matches the base rate of event occurrence in the sample.

\section{Step 2: Decomposition of the correlation-the lens model equation}

The lens model equation (LME) for decomposing the correlation coefficient was developed by Hursch et al. (1964) and has been revised and extended by Tucker (1964), Castellan (1973), Stewart (1976), and Cooksey and Freebody (1985). The LME shows that the correlation is determined by properties of the environmental system, the cognitive system and the relations between them. Stewart (1990) and Lee and Yates (1992) have shown that combining the lens model decomposition with a skill score or MSE decomposition can provide a useful tool for analyzing forecasting skill. In this paper, we expand upon this idea and extend the formulation described by Stewart (1990). 
The lens model equation is based on Brunswik's (1956) lens model (Figure 1). On the left is the observed event, that is, what actually occurred after the forecast was made. On the right is the forecast itself. Intervening between the forecast and the event are the items of information, or 'cues', used to make the forecast.

In this schematic representation of the lens model, the lines connecting the cues and the observed event represent relations between the cues and the observed event in the environmental system. Lines connecting the cues and the forecast represent relations between the cues and the forecast in the cognitive system, that is, how the forecaster uses cues. Lines connecting cues indicate that there are relations among the cues themselves; that is, they are not independent (only a few representative lines have been drawn).

Since the forecast event is not fully determined by the cues, it cannot be predicted with certainty. Similarly, because some inconsistency is pervasive in human judgment, forecasts also are not perfectly related to the cues. Therefore, the relations on both sides of the lens model are probabilistic; that is, there is an element of uncertainty in the relation between the cues and both the observed event and the forecast. This is an example of the parallelism between properties of the environmental and cognitive sides of the lens model that is central to Brunswik's theory (Hammond et al., 1975).

These probabilistic relations can be expressed formally by partitioning variables representing the observed event and the forecast into two components-one that is a function of the cues and another that is unrelated to them. This partitioning can be written as:

$$
\begin{aligned}
& O=M_{O . X}\left(X_{1}, X_{2}, \ldots, X_{n}\right)+E_{O . X} \\
& Y=M_{Y . X}\left(X_{1}, X_{2}, \ldots, X_{n}\right)+E_{Y . X}
\end{aligned}
$$

where the $X_{i}$ are the cues; $M_{O . X}$ and $M_{Y . X}$ represent models that describe the relations between the cues and the event and the cues and the forecast, respectively; and the $E$ 's, which represent the residuals or 'errors' of the models, are not related to the $M$ 's.

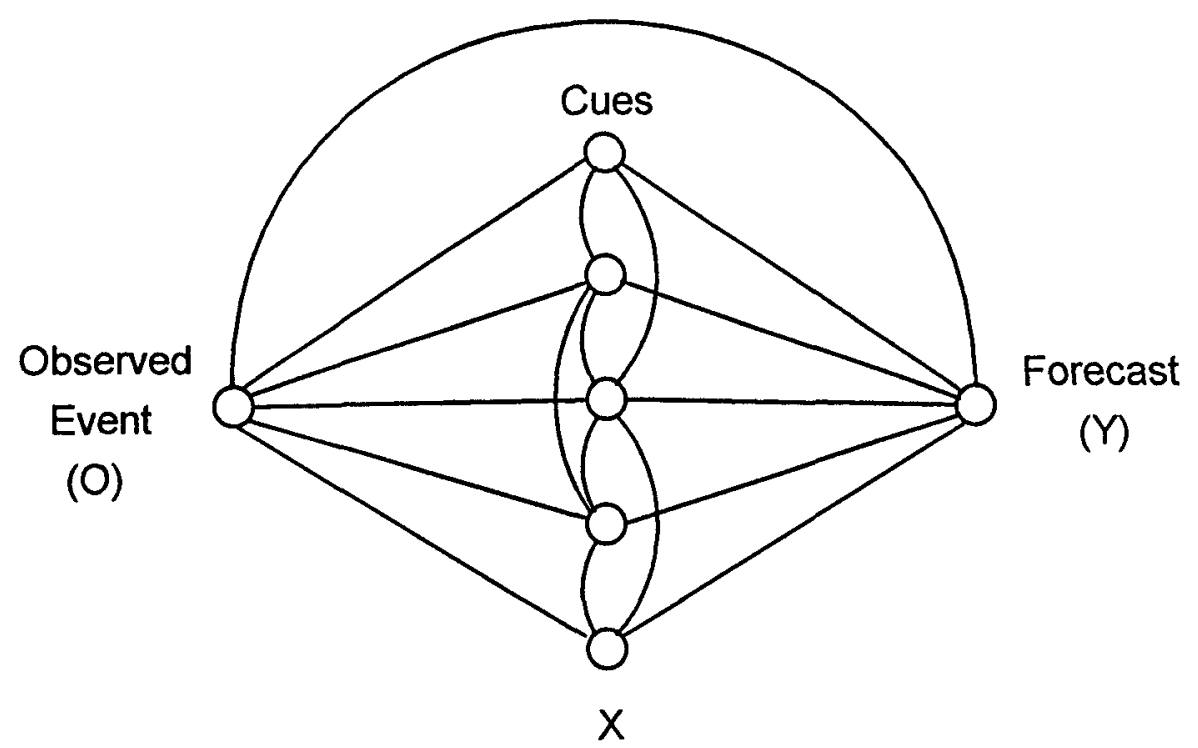

Figure 1. Brunswik's lens model 
This partitioning of the forecast and the observed event can be used to derive a partitioning of the correlation between them (Stewart, 1976). Based on that partitioning, Tucker (1964) developed the following form of the lens model equation:

$$
r_{Y O}=R_{O . X} G R_{Y . X}+C \sqrt{1-R_{O . X}^{2}} \sqrt{1-R_{Y . X}^{2}}
$$

where

$$
\begin{aligned}
& R_{O . X} \text { is the correlation between } O \text { and } M_{O . X}, \\
& G \text { is the correlation between } M_{O . X} \text { and } M_{Y . X}, \\
& R_{Y . X} \text { is the correlation between } Y \text { and } M_{Y . X} \text {, and } \\
& C \text { is the correlation between } E_{Y . X} \text { and } E_{O . X} .
\end{aligned}
$$

If $M_{Y . X}$ captures all the systematic relations between the cues and the forecast, then $E_{Y . X}$ will be unrelated to the outcome event and $C$ will be small. ${ }^{1}$ The following approximation then holds:

$$
r_{Y O} \cong R_{O . X} G R_{Y . X}
$$

This formulation expresses the correlation as the product of three components:

- Ro.x: The strength of the relation between the observed event and the cues. If $M_{o . x}$ is the optimal model of the observed event (that is, it exhausts all the systematic relations between the cues and the observed event), then this component measures the maximum predicability of the observed event for the given set of cues.

- G: The match between the environmental model and the forecast model. This is correlation between (1) the model of the systematic relations between the observed event and the cues, and (2) the model of the forecaster's cue utilization. ${ }^{2}$ In other words, it is a measure of how well the model of the forecast matches the environmental model.

- $R_{Y . X}$ : The strength of the relation between the forecast and the cues. If $M_{Y . X}$ exhausts all the systematic relations between the cues and the forecast, then this component could be considered a measure of the reliability of the forecast. ${ }^{3}$

Step 3: Further decomposition based on expanded lens model

For a given set of cues, environmental predictability could be limited by (1) errors in the cues or (2) imperfect relations between the cues and the observed event. Similarly, forecaster unreliability could be due to (1) unreliability in acquiring cue information or (2) unreliability in organizing that information into a forecast. These concepts form the basis for a further decomposition of environmental predictability and forecast reliability.

Figure 2 presents a version of the lens model that has been expanded by inserting two

\footnotetext{
${ }^{1}$ Although $C$ is usually found to be low in practice, high values are possible. High values of $C$ indicate either that the relation between the cues and the forecast have not been adequately modeled or that one or more cues that are available to the forecaster are not included in the model. In either case, revision of the model is indicated. For discussion of the implications of various values of $C$, see Stewart (1988).

${ }^{2}$ For brevity, we use 'correlations between models' to refer to 'correlations between the outputs of models'. There is no loss of clarity.

${ }^{3}$ Reliability refers to the ability of a measurement procedure to produce the same results if the same objects are measured on repeated occasions. Unreliability is due to random or error variance which may reflect a number of sources of measurement error (Kerlinger, 1973). When the term 'reliability' is used in connection with human judgment, it refers to the extent to which identical information leads to identical judgments, that is, the proportion of systematic or repeatable variance in the judgments. The term 'consistency' is used to refer to the fit of a specific model to a set of judgments. If the model captures all of the systematic variance in the judgments, then consistency with respect to that model is identical to reliability.
} 


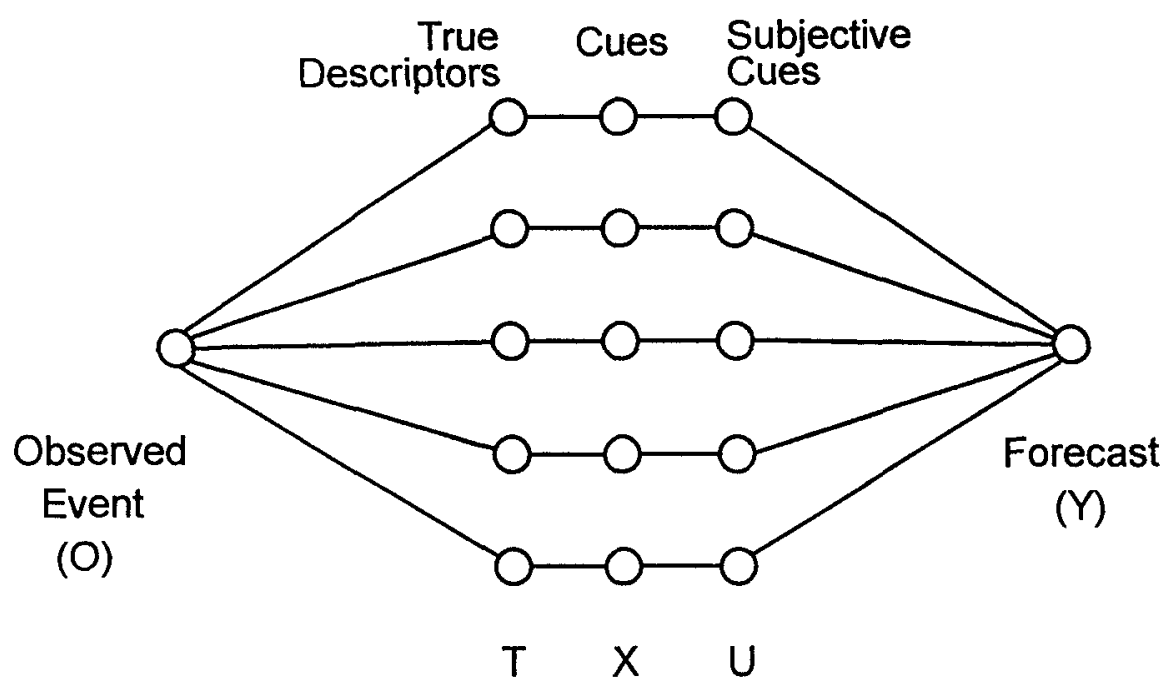

Figure 2. An expanded lens model

additional sets of variables. One set, labeled 'true descriptors', is interposed between the cues and the observed event. These variables represent the 'true' environmental conditions that the observed cues are intended to capture. For example, the windspeed at a ground station might be $24 \mathrm{~m} / \mathrm{s}$, but, due to instrument error or time delays, the reading transmitted to the weather forecaster as a cue might be $22 \mathrm{~m} / \mathrm{s}$. In the case of economic forecasting, an example of a true descriptor would be actual unemployment, while the cue available to the forecaster is an estimate based on a survey. The expanded lens model acknowledges that the cues available to the forecaster often provide an imperfect representation of the true conditions in the environment.

A second set of variables, labeled 'subjective cues', is interposed between the cues and the forecast. These variables represent the subjective data that the forecaster integrates into a forecast. For example, colored images on a radar display might indicate that windspeed in a storm is $24 \mathrm{~m} / \mathrm{s}$, but the weather forecaster might interpret it as $28 \mathrm{~m} / \mathrm{s}$. The forecaster would then base the forecast on the subjective value of $28 \mathrm{~m} / \mathrm{s}$, rather than on the objective data of $24 \mathrm{~m} / \mathrm{s}$. An example of a subjective cue in economic forecasting would be the forecaster's inspection of a time series to detect patterns or cycles. Different forecasters could arrive at differing subjective interpretations of the graph because they use different conceptual models to interpret the data.

\section{Decomposition of environmental predictability}

The expanded lens model indicates that there are two factors that limit predictability of the environment $\left(R_{O . X}\right)$ : (1) imperfect relations between the cues and the true descriptors and (2) imperfect relations between the true descriptors and the observed event. These two components can be formally separated by defining a function, $M_{O . T}$, to represent the systematic functional relations between the event and the true descriptors $\left(T_{i}\right)$ :

$$
O=M_{O . T}\left(T_{1}, T_{2}, \ldots, T_{n}\right)+E_{O . T}
$$

It is assumed that $M_{O . T}$ is not correlated with $E_{O . T}$.

$R_{O . T}$ is defined as the correlation between $O$ and $M_{O . T}$. It is a measure of the predictability 
of the environment, given true descriptors. Since true descriptors are, by definition, not readily available to the forecaster, it will usually be difficult to measure them. Because of the difficulty of measuring the true descriptors, and because the optimal environmental model relating those descriptors to the observed event is generally unknown, it will generally be impossible to obtain a good estimate of $R_{O . T}{ }^{4}$ Therefore, the decomposition of environmental predictability based on the expanded lens model is more useful conceptually than analytically.

The ratio of $R_{O . X}$ to $R_{O . T}$ theoretically lies between 0.0 and 1.0 and represents the effect of measurement error, or lack of fidelity in the information system, on forecast skill. Let $V_{T, X}$ denote that ratio. Then,

$$
R_{O . X}=R_{O . T}\left(\frac{R_{O . X}}{R_{O . T}}\right)=R_{O . T} V_{T . X}
$$

thus decomposing the previous measure of environmental predictability into the product of 'environmental predictability' given true predictors $\left(R_{O . T}\right)$ and 'fidelity of the information system' $\left(V_{T, X}\right)$.

$V_{T, X}^{2}$ is the proportion of valid variance (with respect to $O$ ) in the true descriptors that is maintained in the observed cues, $X_{i}$. Expressions for $V_{T . X}$ depend on the form of $M_{O . T}$ and on the relation between the $T_{i}$ and the $X_{i}$. For example, if $M_{O . T}$ is linear and the $X_{i}$ are obtained by adding random error $\left(E_{i}\right)$ to the $T_{i}$, i.e. $X_{i}=T_{i}+E_{i}$, then it can be shown that ${ }^{5}$

$$
\begin{gathered}
V_{T . X}^{2}=\frac{s_{\hat{O}_{T}}^{2}}{s_{\hat{O}_{T}}^{2}+s_{E_{x}}^{2}} \quad \text { where } \quad \hat{O}_{T}=\sum_{i=1}^{n} b_{i} T_{i}+k \quad \text { and } \quad s_{E_{x}}^{2}=\sum_{i=1}^{n} b_{i}^{2} s_{E_{i}}^{2} \\
\left(b_{i} \text { are least squares regression weights }\right) .
\end{gathered}
$$

Unsurprisingly, the fidelity of the information system decreases as the total error variance in the cues increases relative to the variance of the true-descriptor regression model.

\section{Decomposition of forecast reliability}

The expanded lens model indicates that forecaster reliability is reduced by two factors-imperfect relations between objective cues and subjective cues (unreliability of subjective interpretation of cues ${ }^{6}$ ), and imperfect relations between the subjective cues and the forecast (unreliability of information processing). These two components can be formally separated by defining a function, $M_{Y . U}$, that captures the systematic relations between the subjective cues $\left(U_{i}\right)$ and the forecast:

$$
Y=M_{Y . U}\left(U_{1}, U_{2}, \ldots, U_{n}\right)+E_{Y . U}
$$

$M_{Y . U}$ is assumed to be uncorrelated with $E_{Y . U}$.

$R_{Y . U}$ is defined as the correlation between $Y$ and $M_{Y . U}$. Assuming that $M_{Y . U}$ captures all the systematic variance in the forecast, $R_{Y . U}$ is a measure of the forecaster's informationprocessing reliability.

If the true values of the subjective cues $\left(U_{i}\right)$ were known, then they would, in general, be better predictors of the forecast than the objective cues $\left(X_{i}\right)$ because the objective data are imperfectly related to the subjective cues that are integrated into the forecast. As a result, $R_{Y . X}$

\footnotetext{
${ }^{4}$ In the case of weather forecasting, there have been attempts to estimate true environmental unpredictability (Barnett and Preisendorfer, 1978; Chu and Katz, 1987).

${ }^{5}$ The derivation of this formulation is available from the authors.

${ }^{6}$ Systematic errors in the transformation from objective to subjective cues are also possible. For example, a forecaster could consistently interpret a cue as too high or too low. This type of systematic error could result in an increase in unconditional bias or, if it affected the cue weight, could reduce the match between the forecaster and the environment. We thank Michael Doherty for pointing out this possibility.
} 
will be less than $R_{Y, U}$, and $\left(R_{Y, X} / R_{Y, U}\right)$ represents the effect of unreliability of information acquisition on forecast skill. Let $V_{U . X}$ denote that ratio. Then,

$$
R_{Y . X}=R_{Y . U}\left(\frac{R_{Y . X}}{R_{Y . U}}\right)=R_{Y . U} V_{U . X}
$$

thus decomposing forecast reliability into the product of reliability of information processing $\left(R_{Y . U}\right)$ and the reliability of information acquisition $\left(V_{U . X}\right)$. As was shown for $V_{T . X}$, expressions for $V_{U . X}$ can be derived if assumptions are made about the form of the model relating subjective cues to the forecast and about the nature of the relation between the objective cues and the subjective cues. Assuming a linear $M_{Y . U}$ and $U_{i}$ resulting from adding random error to the $X_{i}$, it can be shown that the reliability of information acquisition decreases as the error variance in the subjective cues increases, relative to the variance in the subjective-cue regression model.

\section{Summary}

In three stages, the skill score has been decomposed into seven components. First, Murphy's decomposition separates bias from the correlation. Second, the lens model equation (with some assumptions) separates the effects of environmental predictability and forecast reliability from the match between cue-event relations and cue-forecast relations. Third, an extension of the lens model equation decomposes both environmental predictability and forecast reliability into two components each. The steps in the decomposition are illustrated in Figure 3.

The first two decompositions are useful for empirical analysis of forecast performance (Stewart, 1990), but the data necessary to estimate all the parameters of the full decomposition

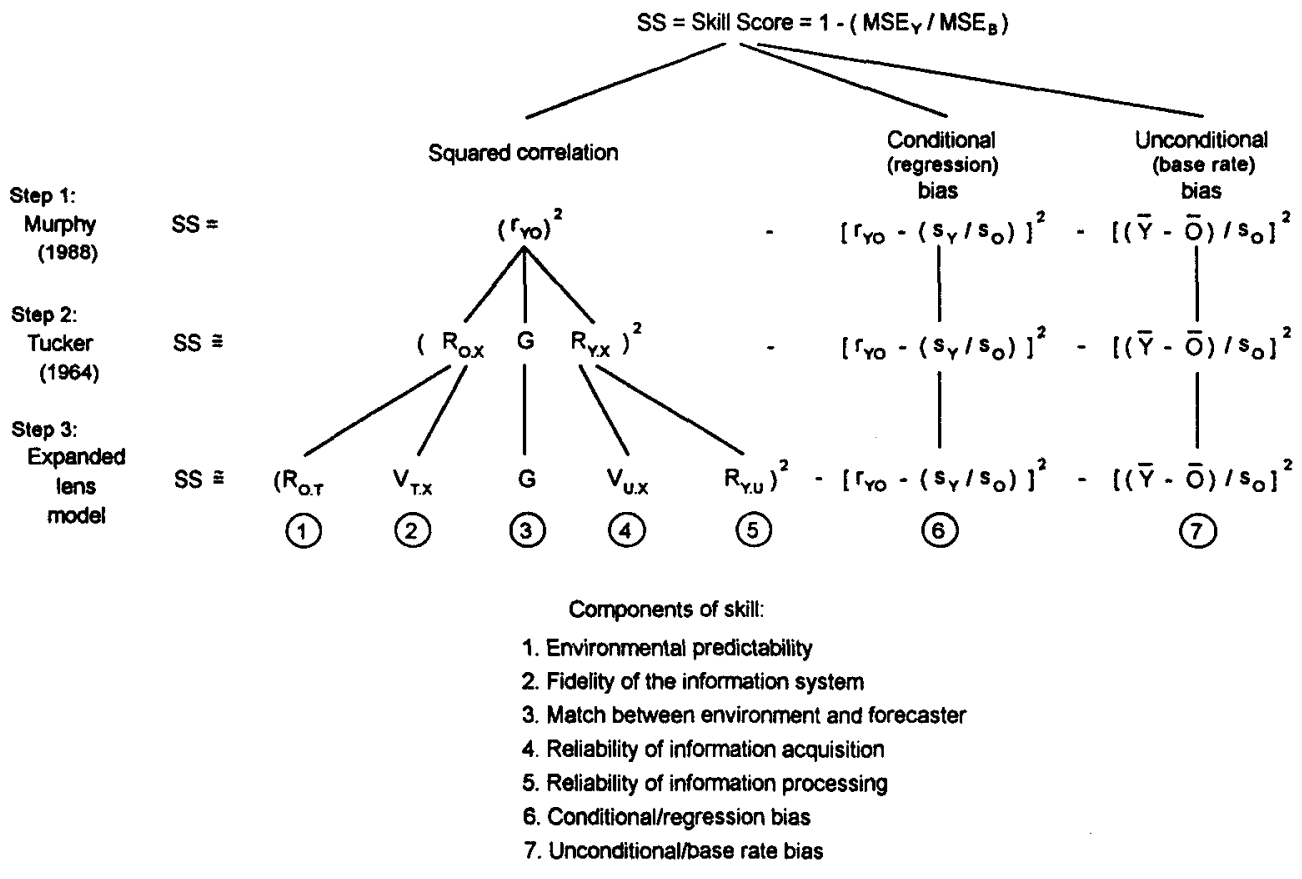

Figure 3. Summary of decomposition equations 
will rarely be available. In particular, obtaining the data necessary to measure fidelity of the information system will be impossible in most field studies. Although it is possible (and, we would argue, desirable) to obtain the measures of subjective cue values necessary to estimate reliability of information acquisition, they rarely are available in studies conducted outside the laboratory. For purposes of this paper, therefore, we emphasize the conceptual and theoretical value of the full decomposition.

\section{IMPLICATIONS FOR IMPROVING PERFORMANCE}

Since the full decomposition is presented for the first time here, no individual study addresses all its elements. Representative research on factors that affect components of skill and methods for improving forecast skill is reviewed in this section. Table I summarizes the second topic-implications of research for improving forecasts.

There is a column in Table I for each component of skill. The rows include both methods for improving skill that have been evaluated in the literature reviewed here and other methods that have not been studied but are included because research and theory suggest that they are worthy of study. Marked cells indicate a close theoretical or empirical match between skill component and method. The greater number of rows in the table associated with columns 3-7

Table I. Components of skill addressed by selected methods for improving forecasts

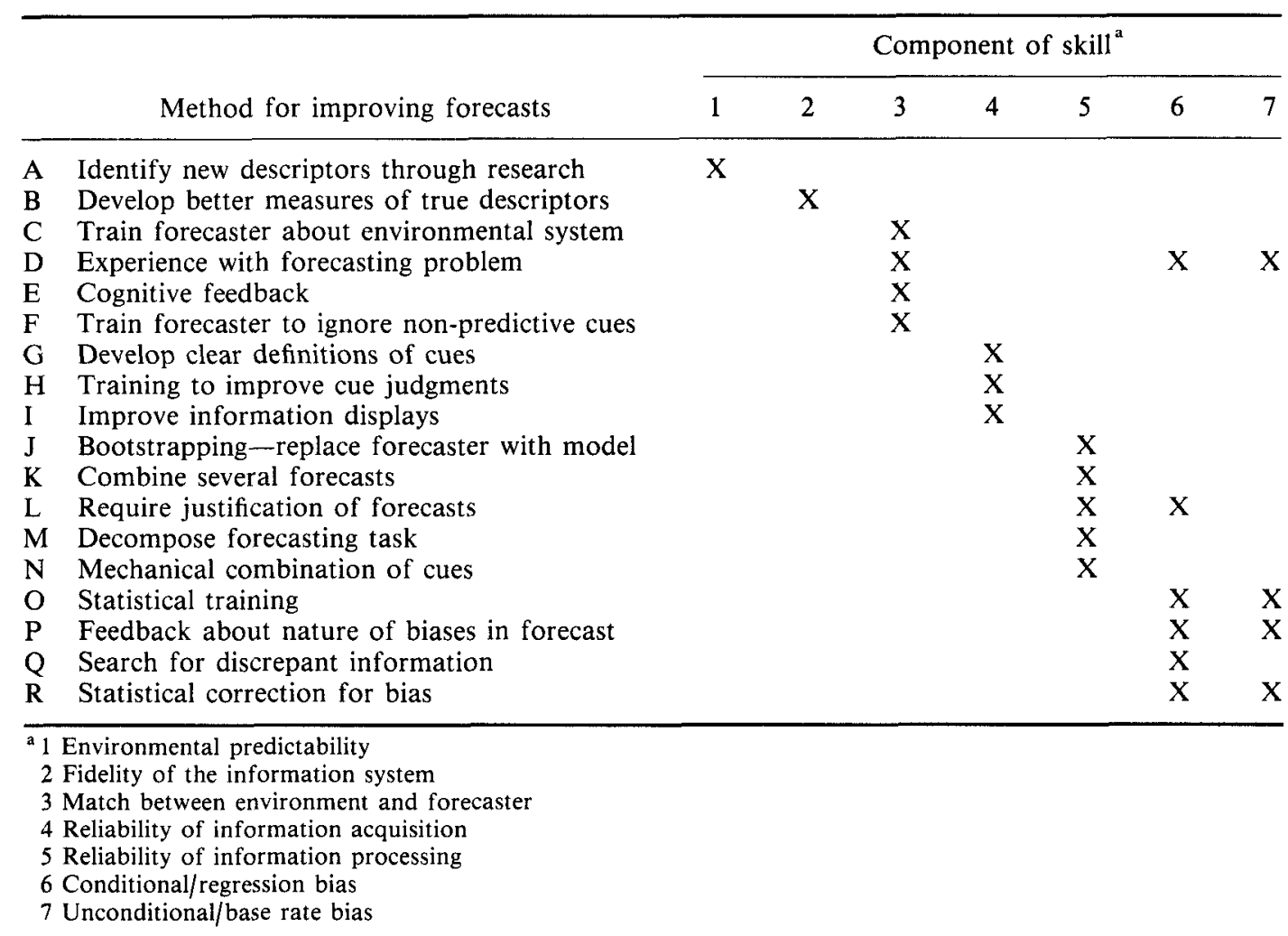


reflects the emphasis in this paper on the components of skill that are fully or partially within the forecaster's control.

Where marked cells in Table I are referred to in the following text, cell references are included in parentheses.

\section{Environmental predictability}

Environmental predictability determines an upper bound on forecast performance and therefore indicates how much improvement is possible through attention to other components. In laboratory studies, tasks with known environmental predictability have been studied (e.g. Armelius, 1979; Hagafors and Brehmer, 1983). Brehmer (1976) found that a decrease in environmental predictability for a laboratory task was associated with a decrease in judgmental consistency.

In field studies, environmental predictability often cannot be estimated with any accuracy. Furthermore, for a given set of information, environmental predictability is fixed and not under the control of the forecaster. It may be possible to improve predictability by identifying valid new descriptors that can be used in forecasting (A1). Since our interest here is in the more subjective aspects of forecasting skill, we will not discuss environmental predictability further.

\section{Fidelity of the information system}

Forecasting skill may be degraded if the information system that brings data to the forecaster does not accurately represent actual conditions, i.e. if the cues do not accurately measure the true descriptors. Fidelity of the information system refers to the quality, not the quantity, of information about the cues that are currently being used.

The available research indicates, surprisingly, that for some tasks, measurement error in cues may not be a major determinant of performance. In a review of forecasting methods for marketing, Armstrong et al. (1987) report that measurement error in the environmental inputs to a market forecasting model had little impact on the accuracy of environmental forecasts. York et al. (1987) investigated the effect of cue unreliability in a multiple cue probability learning (MCPL) task. They compared a group that received 'true scores' to groups that received cue values with random error added to the true score values and concluded that measurement error in the predictor does not affect performance. However, their task was structured so that the five values presented for each cue could be averaged for optimal performance.

The generality of these findings and the reasons for them require further investigation. If the cues available to judges are poor predictors, then more accurate measurement of them might not improve judgment. A more likely explanation is that redundancy in the cues, or multiple measurement of the same cue, cancels out the effect of measurement error. In the case of redundant information, error in measurement of a single cue might have little effect on the predictability of the entire set because fidelity of the information system depends on the ability of all the cues available to the forecaster to recover the valid variation in the true predictors. Therefore, errors in individual cues might not have much effect on overall system fidelity.

Problems with the fidelity of the information system need not be limited to the traditional concept of measurement error. Another kind of error, called system failure error $\left(\mathrm{O}^{\prime}\right.$ Connor et al. 1989) could be more devastating. In this case, all valid information is lost due to, for example, instrument failure. If the forecaster were unaware of system failure, he or she could be seriously misled by the information provided. The urgency of correcting system failure would override all other components of skill until the failed system were repaired or replaced.

Measurement error and accuracy of information is a major concern to forecasters in any 
domain. Designers of information systems for forecasting will naturally seek to maximize the fidelity of the system by obtaining the best possible measures (B2). The problems involved in doing so are domain-specific, and it is likely that they will have been addressed, with varying degrees of success, in situations where important forecasts are made. For example, in the weather domain, Bader et al. (1988) acknowledge that quality control is becoming increasingly important and provide examples of how meteorological data can be cross-checked.

Environmental predictability and fidelity of the information system are components of skill that are determined by the forecast domain, the available information relevant to the forecast, and the information system. They are beyond the control of the forecaster, at least in the short run. We now turn to elements of the decomposition that are directly influenced by the forecaster's behavior.

\section{Match between environment and forecaster}

The match between the model of the forecaster and the environmental model is measured by the correlation $(G)$, over a set of forecasts, between the forecaster model $\left(M_{Y . X}\right)$ and the environmental model $\left(M_{O . X}\right)$. It is an estimate of the potential skill that the forecaster's current strategy could achieve if the environment were perfectly predictable (given the cues) and the forecasts were unbiased and perfectly reliable.

In some applications of the lens model equation, the $G$ parameter has been called 'knowledge' (Hammond et al., 1973; Beal et al., 1978). As Beal et al. (1978) point out, however, $G$ does not represent knowledge in the traditional sense of mastery of a subject area or an intellectual field. Rather, $G$ measures the individual's knowledge of the requirements of the prediction task and the ability to apply that knowledge to forecasting. See Castellan (1992) for an important discussion of $G$ and cautions regarding its use.

An obvious way to improve the environment/forecaster match is to have the forecaster learn more about the nature of the environmental system (C3). Thus, a weather forecaster's performance might be improved by learning more about atmospheric science (a basic premise of National Weather Service training programs), or economic forecasts might be improved by learning more economics. Such learning does not guarantee improved performance, however, because forecasters who are extremely knowledgeable about the environmental system might still have low $G$ 's if they are unable to apply that knowledge to the forecasting task (Gedzelman, 1979).

Increasing the forecaster's experience with the task (D3) is another obvious way to improve $G$. As Brehmer (1980) has argued, the nature of experience and human information-processing biases are such that it is very difficult to learn a complex task from experience alone. If Brehmer's arguments apply to a forecasting problem, then the improvement in $G$ with experience alone could be very slow.

A study conducted by Stael von Holstein (1971) suggested that if experience were accompanied by extensive feedback and discussion of the results, the forecaster-environment match could improve. He conducted a study of probabilistic forecasts of temperature and precipitation, providing feedback regarding accuracy (using a quadratic scoring rule) and the probability distributions, as well as outcome feedback. He did not find a change in accuracy over time. However, he found that research assistants performed better than meteorologists. He argued this was because the research assistants had opportunities to discuss their feedback, whereas the meteorologists received feedback in written form with no discussion. He further suggests that the meteorologists would have benefited from more extensive feedback.

A method that has been found useful in accelerating the rate of learning complex judgment tasks is 'cognitive feedback' (E3) (Hammond, 1971). A review by Balzer et al. (1989) 
distinguishes among three types of cognitive feedback: task information, cognitive information, and functional validity information. Task information includes feedback regarding the task uncertainty, relation between cues and the observed event, and cue intercorrelations. Cognitive information mirrors the task information in terms of how the judge weighs cue information and reliability. Functional validity information includes achievement (i.e. the correlation coefficient), $G$, and $C$. The authors suggest that task information is the most important feedback component and suggest that, if available, it should be used to train judges. Information about the level and variability of the criterion may also be useful task information. They further suggest that cognitive information is of little value. Functional validity information has not been extensively studied.

Wigton et al. (1990) investigated diagnostic judgments of pharyngitis for physicians and medical students. During a six-month intervention they provided cognitive feedback which included learner's weights and the optimal weights, as well as 'probability feedback' (the probability of a positive culture calculated from the optimal cue weights). They found that judgments became better calibrated and more accurate (as measured by the correlation). Matching $(G)$ also increased, but consistency did not. They suggested that increased performance was due to better conformity with the models rather than increased consistency. They also found that students improved more than physicians. The physicians were reluctant to adopt the model's weights.

In contrast to most studies, Tape et al. (1992) found that $G$ increased with outcome feedback (the correct judgment for each case), but not with cognitive feedback. They suggest that the value of cognitive feedback depends upon the task and that it may be more useful when the cue-event relationships 'are complex and the combining rule is not known' (p. 213).

Gaeth and Shanteau (1984) were successful in helping soil judges reduce the influence of irrelevant facts and increase accuracy (F3). They attribute the most success to interactive training'. Such training included not only verbal suggestions for reducing the effect of irrelevant information but also experience (with feedback) with soils containing irrelevant factors. In the medical domain, Wigton (1988) has also suggested that training should include materials designed to discourage reliance on non-predictive cues currently in use.

In summary, the available research suggests that when the environmental model relating cues to observed events is known, information about the model should be provided to forecasters as part of their training. In most cases, the environmental model will not be known, and that is precisely why judgmental forecasting is used. In such cases, it may be possible to estimate a model, or at least distinguish between relevant and irrelevant cues, by analyzing historical data or analyzing the forecasts of established experts. Training and practice with the task might be used to reduce reliance on irrelevant cues. When the environmental model is not known and cannot be estimated, feedback to the judges on the attributes of their judgments, along with a discussion of the results, may enhance performance.

\section{Reliability of information acquisition}

Reliability of information acquisition is the extent to which the forecaster can reliably interpret the objective cues. The evidence suggests that unreliability of information acquisition is pervasive. In a review of research on reliability of clinical judgments in medicine, Koran (1975) found a wide range of intra- and inter-observer reliability in extracting cardiovascular, gastrointestinal and respiratory cues from physical examination. He further reports a range of reliabilities for interpreting diagnostic procedures (e.g. electrocardiography). Einhorn (1974) studied pathologists viewing biopsy slides of cancer patients and reports a wide range of mean intra-judge reliabilities for cues. He also demonstrated a procedure for identifying three cue 
judgment biases: (1) under- or overvaluing signs in general ('leniency error'), (2) the tendency to over- or undervalue particular cases (halo effect), and (3) under- or overvaluing particular signs.

Results of an experiment conducted by Brehmer (1970) indicated that unreliability in judging cues made learning more difficult and had an effect similar to that of unpredictability in the environment. This suggests that unreliability in information acquisition may affect not only the quality of the forecasts, but also forecasters' ability to learn from experience.

Lusk and Hammond (1991) distinguish between 'primary' cues that are directly observable from the presented information and 'secondary' cues that must be extracted from a combination of the primary cue values. In studies comparing presentation of primary and secondary cues, they found more disagreement among weather forecasters' probability judgments in the primary cue condition than in the secondary cue condition, which they suggest was due to differential integration of the primary cue information into secondary cue values. They also found that the degree of disagreement on secondary cue values varied considerably by cue. They suggest that this may have been related to differences in proximity of secondary cues to the primary cues. That is, the differences may be due to the varying degrees of subjectivity involved in making the secondary cue judgments.

A special case of secondary cues are cues that describe future, rather than current, conditions and therefore must themselves be forecasted. The evidence reviewed by Armstrong et al. (1987) indicates that unreliability introduced by integrating information to forecast a cue may not be a serious problem. They reviewed 18 studies comparing conditional econometric forecasts (actual data on the causal variables) and unconditional forecasts (causal variables must be forecasted) and found that ten studies showed that conditional forecasts were less accurate than unconditional forecasts, five showed no difference, and only three studies found greater accuracy for conditional forecasts.

Although the research on reliability of information acquisition has not been extensive, the available studies suggest that it is more likely to be a problem in tasks, such as weather forecasting or medical diagnosis, that require interpretation of images or recognition of complex patterns in data that are distributed over time and/or space. For example, Kerr (1991) reported that, in a recent workshop on frontal analysis, different meteorologists placed the same cold front in a variety of geographic positions. The studies reviewed in this and the previous section suggest that in these kinds of tasks, reliability may be more important than fidelity of the information system in limiting forecasting skill, as well as less costly to correct.

Empirical estimates of unreliability in information acquisition could be obtained by having forecasters make repeated cue judgments from the same data or by having different forecasters judge cues based on the same data. Lusk and Hammond (1991) suggested that identification of specific cues demonstrating high levels of disagreement among forecasters makes it possible to focus on variables with the greatest potential for improving judgment.

Despite its importance to forecasting, we have not found any studies that specifically evaluate methods for improving reliability of information acquisition. There are several general suggestions that deserve study and are included in Table I. Lusk et al. (1990) recommend that clear operational definitions be developed for each cue (G4). Reliability might also be improved through forecaster training focused on troublesome cues (H4) or by designing improved displays, taking into account factors that affect reliability (I4).

\section{Reliability of information processing}

Unreliability in processing subjective information is pervasive in human judgment, and some studies have found that it increases as the predictability of the environment decreases (Brehmer 
and Brehmer, 1988; Camerer, 1981). Unreliability may also increase as the amount of information available for forecasting increases (Stewart et al., 1992; Lee and Yates, 1992). Although additional information could serve to improve the forecaster's understanding of the environmental conditions at the time of the forecast, it also increases the complexity of the forecasting task and may impose a cognitive burden on the forecaster that exceeds human information-processing capacity. It is not surprising, therefore, that a number of studies have found that people use only a subset of available information (Brehmer and Brehmer, 1988) and that the accuracy of forecasts does not increase with increasing information (Armstrong, 1985; Brockhoff, 1984; Lusk and Hammond, 1991).

Indirect evidence that information-processing reliability affects the accuracy of judgments comes from studies showing that simple techniques for increasing reliability result in improved accuracy. One such technique, 'bootstrapping', substitutes the regression model of the forecasts for the original forecasts (J5). Often forecasts based on the perfectly reliable regression model perform better than the original forecasts produced by the less than perfectly reliable human (Armstrong, 1985; Camerer, 1981). Another simple technique for increasing reliability is averaging the forecasts that were made independently by several individuals (K5) (Kelley, 1925; Stroop, 1932). Greater reliability is one of the reasons that group average forecasts generally outperform members of the group (Armstrong, 1985; Stael von Holstein, 1971).

A study by Hagafors and Brehmer (1983) suggests that reliability might increase if the forecaster were asked to verbally justify forecasts (L5). They found that having to justify one's opinion led to higher consistency when no outcome feedback is provided. It is interesting that the effect of justification was higher in low predictability than in high predictability conditions, suggesting an interaction between the benefits of justification and environmental predictability. They also found that outcome feedback reduced consistency. They suggest that subjects use feedback to test hypotheses, and the hypotheses keep changing, resulting in decreased reliability. Without feedback, hypothesis testing cannot occur and reliability increases. York et al. (1987), however, found that outcome feedback does not always reduce reliability. It may be that outcome feedback can provide increased motivation that increases reliability. ${ }^{7}$

Some decision aids have been designed to reduce the cognitive burden on the forecaster and increase reliability. Generally, they involve decomposition of the task into manageable units and the use of computers or an algorithm to assist in processing information (M5, N5) (Dawes, 1979; Einhorn, 1972; Dawes and Corrigan, 1974; Hammond et al., 1977; Brehmer and Joyce, 1988). For example, MacGregor et al. (1988) investigated varying degrees of problem decomposition ranging from no structure to a full algorithm condition where subjects were asked to provide estimates of intermediate quantities and were provided with combination rules. They found, in general, that increased accuracy and reliability across subjects was associated with greater decomposition of the task.

\section{Bias-conditional (regression bias) and unconditional (base rate bias)}

Together, the two bias terms in the decomposition measure forecast 'calibration'. Almost all the research on calibration has addressed probabilistic judgments. We do not know whether these results will generalize to deterministic forecasts.

One of the common findings in research on calibration is overconfidence; that is, people make probability judgments that are more extreme than they should be, given the evidence and their knowledge. Overconfidence (or under confidence) would increase conditional bias.

\footnotetext{
${ }^{7}$ Michael Doherty, personal communication, 1993.
} 
Lichtenstein et al. (1982) report that overconfidence is pervasive and is most extreme with tasks of greater difficulty. They also report that calibration varies with the type of forecast. For example, the calibration of weather forecasters has improved over the years and is continuing to improve, but physicians are not well calibrated. They suggest that some weather forecasters may be well calibrated because, unlike physicians, meteorologists have a lot of practice with probability judgments, the task is always the same, and they receive outcome feedback. This suggests that, for some tasks, experience with the forecasting problem may improve calibration (D6, D7).

Fischhoff (1982) identifies three assumed sources of biases that have been addressed by researchers: faulty tasks, faulty judges, and the mismatch between judges and tasks. He reviewed the literature on overconfidence to determine the extent to which manipulations of each source were successful. He concluded that studies attempting to address faulty tasks (e.g. clarifying instructions and using better response modes) have been generally unsuccessful. Research on faulty judges has been more successful. Fryback (1985) argues that to improve subjective probability assessments, clinicians should keep records of their judgments and receive statistical training $(06,07)$, but he provides no data on the success of this method. Lichtenstein and Fischhoff (1980) provided subjects with feedback regarding their judgments, including calibration curves and measures of overconfidence, calibration, knowledge, and resolution (P6, P7); and they found improvement in calibration without affecting resolution. Murphy and Daan (1984) provided detailed feedback including reliability diagrams and quantitative scores describing calibration, resolution and skill (P6, P7). The nature and implications of this feedback were discussed, emphasizing the need to separate the forecast formulation process from the process of using the forecast as a means of reducing the preference for false alarms over misses. They found improvement in skill due primarily to increases in calibration, with little improvement in resolution. They assert that the lack of improvement in resolution is not surprising since it is limited by the state of the art of weather forecasting.

Regarding the mismatch between judges and the task, encouraging judges to search for discrepant information has resulted in improved calibration (Q6; Q7 is blank because evidence suggests that the search for discrepant information acts mainly on overconfidence). Koriat et al. (1980) had subjects list reasons supporting and/or contradicting responses. They found that listing contradictory reasons improved confidence judgments. They suggest that such judgments are made in two stages, both of which involved bias: (1) knowledge search, which favors positive evidence, and (2) evidence review, during which negative evidence is disregarded and confidence assigned. Arkes et al. (1987) found that subjects who anticipated having to justify their answers to a group demonstrated a drop in confidence and improvement in calibration (L6). Fischhoff and MacGregor (1982) discovered that asking subjects to list reasons that their answers might be correct and/or incorrect had a small, but consistent effect on reducing overconfidence (L6). They suggested that other desirable features of the thought listing procedure might be: (1) providing a record of reasons for one's forecasts in order to avoid hindsight bias once the outcome is known (Fischhoff, 1975), (2) allowing for external review of one's reasoning which might lead to correction of misconceptions (Hogarth and Makridakis, 1981), and (3) helping raise one's alertness to new evidence that should prompt revisions of a forecast.

Another approach to improving calibration is to apply a correction formula to the forecasts when the judge's biases are known (R6, R7). Ahlburg (1984) used Theil's (1966) decomposition to identify sources of error in price expectations and forecasts of new housing starts. $\mathrm{He}$ identified bias and regression components of forecast error and applied a correction to the 
forecasts based on a model of the environment and improved accuracy. Theil (1966) and Stewart and Reagan-Cirincione (1991) have provided formulas for debiasing forecasts. Application of such formulas assumes that the forecaster's biases are stable over time and that the knowledge that forecasts are being adjusted does not, itself, cause the forecaster to change.

The previous research indicates that biases are pervasive and sometimes difficult to overcome. Some evidence suggests that experience with probabilistic judgments reduces bias. Extensive individual feedback on the nature of the biases can decrease biases. Procedures requiring judges' justification of responses and/or consideration of contradictory reasoning can also decrease bias. In addition, it may be possible in some cases to adjust the forecasts to remove bias.

\section{DISCUSSION}

The decomposition we have proposed provides a framework for research on judgmental forecasting and for diagnosing forecasting problems and prescribing methods for improving forecasts. Table I summarizes, with appropriate caveats regarding the lack of adequate research on judgmental forecasting, a number of suggestions for improving forecasts, beginning with the identification of new cues through research. Since poor performance may be due to a ceiling on environmental predictability, rather than to subjective aspects of the forecasting process, attempts to increase the ceiling through research and development are fundamental to the forecasting process. Likewise, sources of error in the information system will limit even the best forecaster's performance. Since the first five components of skill combine multiplicatively, efforts to increase the ceiling on environmental predictability and improve the fidelity of the information system will increase the potential improvement to be gained by attention to the subjective components of the forecast. Unfortunately, in many domains, efforts to improve forecasts are entirely devoted to research and development and to improving the information system because it is assumed that increasing the amount and quality of information is all that is necessary to improve forecasts.

There is probably a point in any forecasting domain where addressing the subjective components becomes more cost effective than further technical research and development. Reliability of information acquisition appears to be an important source of problems, and potential improvement, for those forecasting tasks that rely on judgment processes to acquire information. Surprisingly little research is available on this component. The few available studies provide examples of how to identify cues that are adversely affected by unreliability.

Although the other judgmental components of forecasts have been more thoroughly investigated and several methods for improvement have been suggested, more research is needed to identify which are most likely to cause difficulties in particular forecasting contexts and which can be most effectively improved. Most previous studies of judgmental skill have investigated overall skill or perhaps only one or two of its components. It is quite possible, however, that methods designed to address one component have unintended effects on others. For example, bootstrapping (row J) directly affects reliability of information processing, but it will also decrease the standard deviation of forecasts, possibly influencing conditional bias. Identification of new descriptors (row A) to increase environmental predictability may also decrease reliability of information processing by forcing the forecaster to cope with increasing amounts of information. Because of the possibility of indirect and unanticipated effects of 
methods on skill components, a thorough understanding of forecasting skill requires studies designed to collect data about all relevant components.

\section{APPENDIX: DERIVATION OF AN EXPRESSION FOR $V_{T, X}$ WHEN $M_{O . T}$ IS LINEAR AND RANDOM ERROR IS ADDED TO $T_{i}$ TO OBTAIN $X_{i}$}

Let $\hat{O}_{T}=\sum_{i=1}^{n} b_{i} T_{i}+k$ where the $b_{i}$ are least squares regression weights. The multiple regression coefficient, $R_{O . T}$, is the correlation between $\hat{O}_{T}$ and $O$.

Let $X_{i}=T_{i}+E_{i}$, where $E_{i}$ are random errors, with means of 0.0 , uncorrelated with $T_{i}$ (for all $i$ ) and with $O$. It can then be shown that the least squares regression weights for predicting $O$ from $X_{i}$ are identical to those for predicting $O$ from $T_{i}$ (the easiest way to show this involves the matrix formulation for the regression weights), and, therefore,

$$
\begin{gathered}
\hat{O}_{X}=\sum_{i=1}^{n} b_{i} X_{i}+k \\
\hat{O}_{X}=\sum_{i=1}^{n} b_{i}\left(T_{i}+E_{i}\right)+k=\sum_{i=1}^{n} b_{i} T_{i}+\sum_{i=1}^{n} b_{i} E_{i}+k=\hat{O}_{T}-k+E_{X}+k=\hat{O}_{T}+E_{X}
\end{gathered}
$$

where

$$
\hat{O}_{T}=\sum_{i=1}^{n} b_{i} T_{i}+k \quad \text { and } \quad E_{X}=\sum_{i=1}^{n} b_{i} E_{i}
$$

Therefore,

$$
\operatorname{Cov}\left(\hat{O}_{X}, O\right)=\operatorname{Cov}\left(\hat{O}_{T}, O\right)+\operatorname{Cov}\left(E_{X}, O\right)
$$

Since $E_{X}$ and $O$ are uncorrelated,

$$
\begin{gathered}
\operatorname{Cov}\left(\hat{O}_{X}, O\right)=\operatorname{Cov}\left(\hat{O}_{T}, O\right) . \\
R_{O . X}=r_{\hat{O}_{x} . O}=\frac{\operatorname{COv}\left(\hat{O}_{X}, O\right)}{s \delta_{x} s_{O}}=\frac{\operatorname{Cov}\left(\hat{O}_{T}, O\right)}{s \hat{O}_{x} s_{O}} \\
s \hat{O}_{x}=\sqrt{s_{\delta_{T}}^{2}+s_{E_{i}}^{2}} \\
R_{O . X}=\frac{\operatorname{Cov}\left(\hat{O}_{T}, O\right)}{s_{O} \sqrt{s_{\hat{O}_{T}}^{2}+s_{E_{i}}^{2}}} \\
R_{O . T}=\frac{\operatorname{Cov}\left(\hat{O}_{T}, O\right)}{s \hat{O}_{T} s_{O}}
\end{gathered}
$$

Therefore

$$
V_{T . X}=\frac{R_{O . X}}{R_{O . T}}=\frac{s_{\hat{O}_{T}}}{\sqrt{s_{\delta_{T}}^{2}+s_{E_{X}}^{2}}}
$$

where

$$
s_{E_{x}}^{2}=\sum_{i=1}^{n} b_{i}^{2} s_{E_{i}}^{2}
$$




\section{ACKNOWLEDGEMENTS}

Preparation of this paper was supported in part by the National Science Foundation (Division of Social and Economic Sciences and Division of Atmospheric Sciences) under Grants No. SES-8822319 and SES-9109594. We are grateful to Suzanne Wissel for editing the manuscript.

\section{REFERENCES}

Ahlburg, D. A. 'Forecast evaluation and improvement using Thiel's decomposition', Journal of Forecasting, 3 (1984) 345-51.

Arkes, H. R., Christensen, C., Lai, C. and Blumer, C., 'Two methods of reducing overconfidence', Organizational Behavior and Human Decision Processes, 39 (1987), 133-44.

Armelius, K. 'Task predictability and performance as determinants of confidence in multiple-cue judgments', Scandinavian Journal of Psychology, 20 (1979), 19-25.

Armstrong, J. S. Long Range Forecasting, 2nd edition, New York: Wiley, 1985.

Armstrong, J. S., Brodie, R. J. and McIntyre, S. H. 'Forecasting methods for marketing: Review of empirical research', International Journal of Forecasting, 3 (1987), 355-76.

Bader, M. J., Browning, K. A., Forbes, G. S., Oliver, V. J. and Schlatter, T. W., 'Towards improved subjective interpretation of satellite and radar imagery in weather forecasting: Results of a workshop', Bulletin of the American Meteorological Society, 69 (1988), 764-9.

Balzer, W. K., Doherty, M. E. and O'Connor, R., Jr, 'Effects of cognitive feedback on performance', Psycholoical Bulletin, 106 (1989), 410-33.

Barnett, T. P. and Preisendorfer, R. W. 'Multifield analogy prediction of short-term climate fluctuations using a climate state vector', Journal of Atmospheric Science, 35 (1978), 1771-87.

Beal, D., Gillis, J. S. and Stewart, T. R., 'The lens model: Computational procedures and applications', Perceptual and Motor Skills, Monograph Supplement 1, 46 (1978), 3-28.

Brehmer, A. and Brehmer, B., 'What have we learned about human judgment from thirty years of policy capturing?' in Brehmer, B. and Joyce, C. R. B. (eds), Human Judgment: The Social Judgment Theory View, Amsterdam: North-Holland, 1988.

Brehmer, B., 'Inference behavior in a situation where the cues are not reliably perceived', Organizational Behavior and Human Decision Processes, 5 (1970), 330-47.

Brehmer, B., 'Note on the relation between clinical judgment and the formal characteristics of clinical tasks', Psychological Bulletin, 83 (1976), 778-82.

Brehmer, B., 'In one word: Not from experience', Acta Psychologica, 45 (1980), 223-41.

Brehmer, B. and Joyce, C. R. B. (eds), Human Judgment: The Social Judgment Theory View, Amsterdam: North-Holland, 1988.

Brockhoff, K. 'Forecasting quality and information', Journal of Forecasting, 3 (1984), 417-28.

Brunswik, E. Perception and the Representative Design of Psychological Experiments, 2nd edition, Berkeley: University of California Press, 1956.

Camerer, C., 'General conditions for the success of bootstrapping models', Organizational Behavior and Human Decision Processes, 27 (1981), 411-22.

Castellan, N. J., 'Comments on the "lens model" equation and the analysis of multiple cue judgment tasks', Psychometrika, 38 (1973), 87-100.

Castellan, N. J., Jr, 'Relations between linear models: Implications for the lens models', Organizational Behavior and Human Decision Processes, 51 (1992), 364-81.

Chu, P.-S. and Katz, R. W. 'Measures of predictability with applications to the Southern Oscillation', Monthly Weather Review, 115 (1987), 1542-49.

Cooksey, R. W. and Freebody, P., 'Generalized multivariate lens model analysis for complex human inference tasks', Organizational Behavior and Human Decision Processes, 35 (1985), 46-72.

Cronbach, L. J., "Processes affecting scores on "undestanding of others" and "assumed similarity", Psychological Bullein, 52 (1955), 177-93.

Dawes, R. M. 'The robust beauty of improper linear models in decision making', American Psychologist, 7 (1979), 571-82.

Dawes, R. M. and Corrigan, B., 'Linear models in decision making', Psychological Bulletin, 81 (1974), 95-106 
Einhorn, H. J., 'Expert measurement and mechanical combination', Organizational Behavior and Human Decision Processes, 7 (1972), 86-106.

Einhorn, H. J., 'Expert judgment: Some necessary conditions and an examples', Journal of Applied Psychology, 59 (1974), 562-71.

Fischhoff, B. 'Hindsight $\neq$ foresight: The effect of outcome knowledge on judgment under uncertainty', Journal of Experimental Psychology: Human Perception and Decision Processes, 1 (1975), 288-99.

Fischhoff, B., 'Debiasing', In Kahneman, D., Slovic, P. and Tversky, A. (eds), Judgment under Uncertainty: Heuristics and biases, New York: Cambridge University Press, 1982.

Fischhoff, B. and MacGregor, D. 'Subjective confidence in forecasts', Journal of Forecasting, 1 (1982), $155-72$.

Fryback, D. B., 'Decision maker, quantify thyself!', Medical Decision Making, 5 (1985), 51-60.

Gaeth, G. J. and Shanteau, J. 'Reducing the influence of irrelevant information on experienced decision makers', Organizational Behavior and Human Decision Processes, 33 (1984), 263-82.

Gedzelman, S. D., "Reply to "Comment on forecasting skill of beginners" and "Rebuttal to forecasting skill of beginners"', Bulletin of the American Meteorological Society, 60 (1979), 1208-9.

Hagafors, R. and Brehmer, B., 'Does having to justify one's judgments change the nature of the judgment process?' Organizational Behavior and Human Decision Processes, 31 (1983), 223-32.

Hamnmond, K. R., 'Computer graphics as an aid to learning', Science, 172 (1971), 903-8.

Hammond, K. R., Summers, D. A. and Deane, D. H. 'Negative effects of outcome-feedback in multiplecue probability learning', Organizational Behavior and Human Decision Processes, 9 (1973), 30-34.

Hammond, K. R., Stewart, T. R., Brehmer, B. and Steinman, D. O., 'Social judgment theory', in Kaplan, M. F. and Schwartz, S. (eds), Human Judgment and Decision Processes, New York: Academic Press, 1975.

Hammond, K. R., Rohrbaugh, J., Mumpower, J. and Adelman, L., 'Social judgment theory: Applications in policy formation', in Kaplan, M. F. and Schwartz, S. (eds), Human Judgment and Decision Processes in Applied Settings, New York: Acaemic Press, 1977.

Harvey, L. O., Jr, Hammond, K. R., Lusk, C. M. and Mross, E. F., 'The application of signal detection theory to weather forecastirg behavior', Monthly Weather Review, 120 (1992), 863-83.

Hogarth, R. M. and Makridakis, S., 'Forecasting and planning: An evaluation', Management Science, 27 (1981), 115-38.

Hursch, C. J., Hammond, K. R. and Hursch, J. L., 'Some methodological considerations in multiple-cue probability studies', Psychological Review, 71 (1964), 42-60.

Kelley, T. L., 'The applicability of the Spearman-Brown formula for the measrement of reliability', Journal of Educational Psychology, 16 (1925), 300-03.

Kerlinger, F. N., Foundations of Behavioral Research, 2nd edition, New York: Holt, Rinehart and Winston, 1973.

Kerr, R. A., 'A frontal attack on a paradigm of meteorology', Science, 254 (1991), 1591-2.

Koran, L. M., 'The reliability of clinical methods, data, and judgments', New England Journal of Medicine, 293 (1975), 642-6, 695-701.

Koriat, A., Lichtenstein, S. and Fischhoff, B. 'Reasons for confidence', Journal of Experimental Psychology: Human Learning and Memory, 6 (1980), 107-18.

Lee, J.-W. and Yates, J. F. 'How quantity judgment changes as the number of cues increases: An analytical framework and review', Psychological Bulletin, 112, 363-77.

Lichtenstein, S. and Fischhoff, B., 'Training for calibration', Organizational Behavior and Human Decision Processes, 26 (1980), 149-71.

Lichtenstein, S., Fischhoff, B. and Phillips, L. D., 'Calibration of probabilities: The state of the art to 1980', in Kahneman, D., Slovic, P. and Tversky, A. (eds), Judgment under Uncertainty: Heuristics and Biases, New York: Cambridge University Press, 1982.

Lusk, C. M. and Hammond, K. R. 'Judgment in a dynamic task: Microburst forecasting', Journal of Behavioral Decision Making, 4 (1991), 55-73.

Lusk, C. M., Stewart, T. R., Hammond, K. R. and Potts, R. J., 'Judgment and decision making in dynamic tasks: The case of forecasting the microburst', Weather and Forecasting, 5 (1990), 627-39.

MacGregor, D., Lichtenstein, S. and Slovic, P. 'Structuring knowledge retrieval: An analysis of decomposed quantitative judgments', Organizational Behavior and Human Decision Processes, 42 (1988), 303-23.

Murphy, A. H., 'Scalar and vector partitions of the probability score: part I. The two-state situation', Journal of Applied Meteorology, 11 (1972a), 273-82. 
Murphy, A. H., 'Scalar and vector partitions of the probability score: part II. The $N$-state situation', Journal of Applied Meteorology, 11, (1972b), 1183-92.

Murphy, A. H., 'A new vector partition of the probability score', Journal of Applied Meteorology, 12, 595-600.

Murphy, A. H., 'Skill scores based on the mean square error and their relationships to the correlation coefficient', Monthly Weather Review, 116 (1988), 2417-24.

Murphy, A. H. and Daan, H., 'Impacts of feedback and experience on the quality of subjective probability forecasts: Comparison of results from the first and second years of the Zerikzee experiment', Monthly Weather Review, 112 (1984), 413-23.

O'Connor, R., Doherty, M. E. and Tweney, R. D., 'The effects of system failure error on predictions', Organizational Behavior and Human Decision Processes, 44 (1989), 1-11.

Sanders, F., 'On subjective probability forecasting', Journal of Applied Meteorology, 2, (1963), 191-201.

Stael von Holstein, C.-A., 'An experiment in probabilistic weather forecasting', Journal of Applied Meteorology 10, 635-645.

Stewart, T. R., 'Components of correlations and extensions of the lens model equation', Psychometrika, 41 (1976), 101-20.

Stewart, T. R., 'Judgment analysis: Procedures', in Brehmer, B. and Joyce, C. R. B. (eds), Human Judgment: The Social Judgment Theory View, Amsterdam: North-Holland, 1988.

Stewart, T. R., 'A decomposition of the correlation coefficient and its use in analyzing forecasting skill', Weather and Forecasting, 5, (1990), 661-6.

Stewart, T. R. and Reagan-Cirincione, P. 'Coefficients for debasing forecasts', Monthly Weather Review, 119 (1991), 2047-51.

Stewart, T. R., Moninger, W. R., Heideman, K. F. and Reagan-Cirincione, P., 'Effects of improved information on the components of skill in weather forecasting', Organizational Behavior and Human Decision Processes, 53 (1992), 107-4.

Stroop, J. R. 'Is the judgment of the group better than that of the average member of the group?' Journal of Experimenal Psychology, 15 (1932), 550-62.

Tape, T. G., Kripal, J. and Wigton, R. S., 'Comparing methods of learning clinical prediction from case simulations', Medical Decision Making, 12 (1992), 213-21.

Theil, H., Applied Economic Forecasing, Amsterdam: North-Holland, 1966.

Tucker, L. R., 'A suggested alternative formulation in the developments by Hursch, Hammond, and Hursch, and by Hammond, Hursch, and Todd', Psychological Review, 71 (1966), 528-30.

Wigton, R. S., 'Applications of judgment analysis and cognitive feedback to medicine', in Brehmer, B. and Joyce, C. R. B. (eds), Human Judgment: The Social Judgment Theory View, Amsterdam: NorthHolland, 1988.

Wigton, R. S., Poses, R. M., Collins, M. and Cebul, R. D., 'Teaching old dogs new tricks: Using cognitive feedback to improve physicians' diagnostic judgments on simulated cases', Academic Medicine, 65 (1990), S5-S6.

Yates, J. F., 'External correspondence: Decompositions of the mean probability score', Organizational Behavior and Human Decision Processes, 30 (1982), 132-56.

Yates, J. F. and Curley, S., 'Conditional distribution analyses of probabilistic forecasts', Journal of Forecasting, 4 (1985), 61-73.

York, K. M., Doherty, M. E. and Kamouri, J., 'The influence of cue unreliability on judgment in a multiple-cue probability learning task', Organizational Behavior and Human Decision Processes, 39 (1987), 303-17.

\section{Authors' biographies:}

Thomas R. Stewart is Director for Research at the Center for Policy Research, University at Albany, State University of New York, and Research Associate Professor in the Graduate School of Public Affairs, Nelson A. Rockefeller College of Public Affairs and Policy. His recent research focuses on the effects of increasing amounts of information on judgmental forecasts.

Cynthia M. Lusk is currently a research associate at the Cooperative Institute for Research in the Environmental Sciences, and she is also affiliated with the Center for Research on Judgment and Policy, 
both at the University of Colorado. Her recent research concerns judgment and decision-making processes in weather forecasting.

Authors' addresses:

Thomas R. Stewart, Centre for Policy Research, Milne 300, University at Albany, State University of New York, Albany, NY 12222, U.S.A.

Cynthia M. Lusk, Centre for Research on Judgment and Policy, Campus Box 344, University of Colorado, 80309-344, U.S.A. 